\title{
Study of Dielectric Properties of Nano-crystalline Mn-Zn Ferrite
}

\author{
Sunita Bhagwat*, Pratibha Rao \\ Department of Physics, Abasaheb Garware College, Karve Road, Pune - 411 004, India
}

\begin{abstract}
The ac conductivity $\sigma$ and dielectric properties (dielectric constant $\varepsilon^{\prime}$, dielectric loss $\varepsilon$ ' and loss tangent tan $\delta$ ) were measured as a function of frequency between $100 \mathrm{~Hz}$ and $20 \mathrm{MHz}$ for the mixed ferrites $\mathrm{Zn}_{x} \mathrm{Mn}_{1-x} \mathrm{Fe}_{2} \mathrm{O}_{4}$ (where $x=0.2$ to 0.8 ), synthesized by solid-state route. The ac conductivity $\sigma$ below $1 \mathrm{MHz}$ seems to be nearly frequency independent for all compositions; and above these frequency ranges, $\sigma$ sharply increases until it reaches a maximum value. The relaxation time $(\tau)$ was found to be in the range $0.3 \times 10^{-5}-$ $1.5 \times 10^{-5} \mathrm{sec}$ from the hopping frequency. The dielectric constant for these ferrites is approximately inversely proportional to the square root of the resistivity. The electrical conduction mechanism was explained in terms of the electron hopping model. The formation of spinel structure and crystalline size was confirmed using X-ray diffraction (XRD) technique. Morphology of the samples was studied using Scanning Electron Microscopy (SEM).
\end{abstract}

Keywords: Solid-state route; spinel ferrite; dielectric properties; ac conductivity

\section{INTRODUCTION}

Polycrystalline spinel ferrites are known for their sensing applications [1-2] apart from their ever increasing demand in the technological applications in microwave and radio wave devices. These ferrites are widely used for manufacturing transformers and inductors in power supplies [3]. They are mainly classified in two categories: the high permeability ferrites which are used for filtering applications and the low core loss ferrites used for power transformers and inductors in switching mode power supplies. Mn-Zn ferrites are known for magnetic sensors [4]. However, they have not been explored much for microwave applications. The ferrite resonator antennas (FRAs) have the ability to change their characteristics as a function of the applied magnetic field. The low dielectric behaviour makes ferrite materials useful in high frequency applications. Some of the properties of dielectric resonator antennas (DRAs) can be actively controlled using low-loss ferrite materials [5].

In the present work polycrystalline samples of $\mathrm{Zn}_{\mathrm{x}} \mathrm{Mn}_{1-\mathrm{x}} \mathrm{Fe}_{2} \mathrm{O}_{4}$ (x ranges from 0.2 to 0.8 ) were prepared by solid-state route. The structural characterization was studied using XRD. The morphological studies were carried out using SEM.

\subsection{Synthesis Technique}

\section{EXPERIMENTAL}

Polycrystalline samples of $\mathrm{Zn}_{\mathrm{x}} \mathrm{Mn}_{1-\mathrm{x}} \mathrm{Fe}_{2} \mathrm{O}_{4}$ (x ranges from 0.2 to 0.8 ) were prepared by solid-state route using high purity $\mathrm{ZnO}, \mathrm{Fe}_{2} \mathrm{O}_{3}$ and $\mathrm{MnCO}_{3}$ respectively in stoichiometric proportions. The $\mathrm{ZnO} / \mathrm{Fe}_{2} \mathrm{O}_{3}$ and $\mathrm{MnCO}_{3} / \mathrm{Fe}_{2} \mathrm{O}_{3}$ powders were grounded separately for $30 \mathrm{~min}$ using an agate mortar. Each mixture was transferred to a ball mill for $6 \mathrm{~h}$ using $50 \mathrm{ml}$ vials and $12 \mathrm{~mm}$ diameter balls made of stainless steel and a ball-topowder mass ratio of 20. A rotation speed of $240 \mathrm{rpm}$ was used. The milling was done in air atmosphere without any additives. The powder is calcinated at $1000^{\circ} \mathrm{C}$ for $1 \mathrm{~h}$ with a heating rate of $2^{\circ} \mathrm{C} / \mathrm{min}$ followed by cooling at room temperature in air. The pre-sintered samples were crushed again and mixed for another $30 \mathrm{~min}$. with acetone, to be reduced into small crystallites of uniform size. The mixture was air dried and a proper proportion of each powder was again ball milled by adding few drops of 2-propanol. The milling was done with the intention of producing a homogenous mixture of both solid components, without provoking significant structural damage. Finally, the milled reactive powders were dried at $80^{\circ} \mathrm{C}$ in a furnace for $30 \mathrm{~min}$. The powder was compressed uniaxially under a pressure of $1 \times 10^{7} \mathrm{~N} / \mathrm{m}^{2}$ into pellet form of diameter $10 \mathrm{~mm}$ and thickness about $2 \mathrm{~mm}$. The pellets were sintered at $1000^{\circ} \mathrm{C}$ for $3 \mathrm{~h}$ in air atmosphere and cooled to room temperature to make them rigid and impart ceramic properties.

\subsection{Characterization Techniques}

The structural characterization of the ferrite samples was carried out using Philips diffractometer, with $\mathrm{CuK}_{\alpha}$ radiation. The average crystallite size, $\mathrm{t}$, was determined using the Scherrer formula [6]:

$$
\mathrm{t}=0.9 \lambda / \mathrm{B}_{1 / 2} \cos \theta_{\mathrm{b}}
$$

where $B_{1 / 2}$ is the angular line width at half maximum intensity and $\theta_{b}$ is the Bragg angle for the actual peak. 
Dielectric measurements were carried out at room temperature using 4192A impedance analyzer over a wide range of frequency from $100 \mathrm{~Hz}$ up to $20 \mathrm{MHz}$. The dielectric constant $\varepsilon$ ' were calculated using the formula:

$$
\varepsilon^{\prime}=\mathrm{Cd} / \varepsilon_{0} \mathrm{~A}
$$

where $\varepsilon_{0}$ the permittivity of the free space, $C$ is the capacitance, $d$ the thickness and $A$ the cross-sectional area of the pellet.

The dielectric loss factor $\varepsilon$ " has been calculated from the relation:

$$
\varepsilon^{\prime \prime}=\varepsilon ' \tan \delta
$$

where $\tan \delta$ is the loss tangent.

The ac conductivity $\sigma$ of the sample was determined from dielectric parameters using the relation

$$
\sigma_{\mathrm{ac}}=\omega \varepsilon_{\mathrm{o}} \varepsilon^{\prime \prime}
$$

where $\omega$ is the angular frequency.

\section{RESULTS AND DISCUSSION}

\subsection{X-ray diffraction Studies}

Fig. 1 shows the typical X-ray diffraction pattern for $\mathrm{Zn}_{\mathrm{x}} \mathrm{Mn}_{1-\mathrm{x}} \mathrm{Fe}_{2} \mathrm{O}_{4}$ compact disk sintered at $1000^{\circ} \mathrm{C}$ in air for $3 \mathrm{~h}$. The XRD pattern exhibits all the peaks indicative of single phase spinel crystal structure. The nonoccurrence of the other phases suggests there is no solubility limit to $\mathrm{Zn}^{2+}$ ions in the manganese ferrite spinel lattice. It is known that the degree of replacement of the host cations by the other ions in the host lattice depends on the cation radius of the substituent [7]. The crystallite size of all the samples was calculated using the Scherrer formula and it was found to be between 40 and $50 \mathrm{~nm}$.

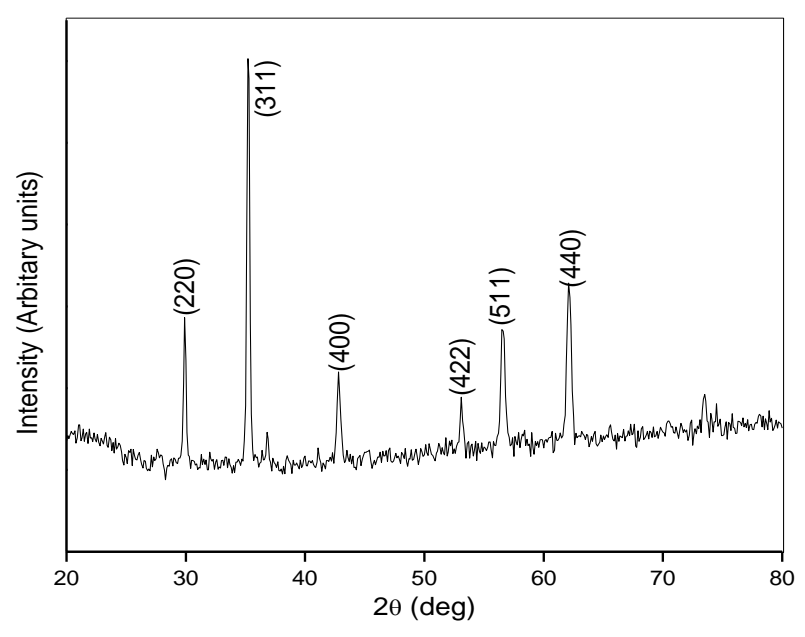

Figure 1: Typical XRD pattern of $\mathrm{Zn}_{\mathrm{x}} \mathrm{Mn}_{1-\mathrm{x}} \mathrm{Fe}_{2} \mathrm{O}_{4}$

\subsection{Dielectric Constant Behaviour}

The dielectric behaviour of ferrite nanoparticles are influenced mainly by the synthesis technique, grain size, cation distribution etc [8]. The behavior of both $\varepsilon^{\prime}, \varepsilon$ '” and $\sigma$ at RT versus frequency are shown in Fig.2 (ae). The general trend for all composition is that $\varepsilon^{\prime}$ and $\varepsilon$ '' decrease with increasing frequency. The decrease in dielectric constant with increasing frequency is attributed to the fact that electron exchange between $\mathrm{Fe}^{2+}$ and $\mathrm{Fe}^{3+}$ ions cannot follow the change of the external applied field beyond certain frequency [9]. At lower frequencies the grain boundaries are more effective than grain electrical conduction. This behavior of a dielectric constant, with $\mathrm{Zn}$ ions substitution can be explained qualitatively on the basis of the mechanism of the polarization process in ferrite which is similar to that in the conduction process. The whole polarization in ferrites is mainly contributed by the space charge polarization, which is governed by the number of space charge carriers. The conductivity in the material and the hopping exchange of charges between two localized states governed by density of the localized state and resultant displacement of charges w.r.t. the external field. As the frequency increases ionic and orientation sources of polarizability decrease and finally disappear due to inertia of the molecules and ions. The high value of the dielectric constant in the present ferrite for $\mathrm{x}=0.2$ may be attributed to space charge polarization [10]; or it could be due to an increase in crystallite size and morphologically clean microstructure as seen in Fig. 3(a). 
An examination of Table I reveals that the values of dielectric constant $\varepsilon^{\prime}$, dielectric loss $\varepsilon$ "' and loss $\operatorname{tangent} \tan \delta$ are maximum for $\mathrm{x}=0.2$. It also can be seen that among these mixed $\mathrm{Mn}-\mathrm{Zn}$ ferrites, the one with the compositions $\mathrm{Zn}_{0.8} \mathrm{Mn}_{0.2} \mathrm{Fe}_{2} \mathrm{O}_{4}$ exhibits the lowest dielectric constant, the lowest dielectric loss, the lowest loss tangent and the lowest electrical conductivity. This might be due the presence of macro-agglomerations containing very fine particles which can be clearly seen in Fig. 3(b).

From Table I it can be seen that the composition $\mathrm{Zn}_{0.2} \mathrm{Mn}_{0.8} \mathrm{Fe}_{3} \mathrm{O}_{4}$ has the maximum dielectric constant (47.9). This high value can be explained as it has maximum number of ferrous ions whose exchange between $\mathrm{Fe}^{2+}$ and $\mathrm{Fe}^{3+}$ gives rise to maximum dielectric polarization, that could be due to the availability of ferrous ions on octahedral B - site. It is also pertinent to mention that the variation of electrical conductivity runs parallel to the variation in dielectric constant as shown in Fig.2. Thus, it is the number of ferrous ions on octahedral sites that play a dominant role in the processes of conduction as well as dielectric polarization. The electrical conduction mechanism can be explained by the electron hopping model of Heikes and Johnston [11]. One obtains local displacements of electrons in the direction of the applied electric field; these displacements determine the polarization of the ferrite.

Table I. Dielectric data of $\mathrm{Zn}_{\mathrm{x}} \mathrm{Mn}_{1-\mathrm{x}} \mathrm{Fe}_{2} \mathrm{O}_{4}$ at $100 \mathrm{kHz}$

\begin{tabular}{|c|c|c|c|c|c|c|c|}
\hline $\mathbf{x}$ & $\boldsymbol{\varepsilon}^{\prime}$ & $\boldsymbol{\varepsilon}^{\prime \prime}$ & $\boldsymbol{\sigma}[/ \boldsymbol{\Omega}-\mathbf{c m}]$ & $\tan \boldsymbol{\delta}$ & $\boldsymbol{\rho} .[\mathbf{\Omega}-\mathbf{c m}]$ & $\operatorname{sqrt}(\boldsymbol{\rho})$ & $\boldsymbol{\varepsilon}^{\prime} * \mathbf{s q r t}(\boldsymbol{\rho})$ \\
\hline 0.2 & $4.79 \mathrm{E}+01$ & $8.82 \mathrm{E}+01$ & $5.04 \mathrm{E}-06$ & $1.84 \mathrm{E}+00$ & $1.98 \mathrm{E}+05$ & 445.4354 & $2.13 \mathrm{E}+04$ \\
\hline 0.35 & $1.27 \mathrm{E}+01$ & $1.06 \mathrm{E}+01$ & $6.06 \mathrm{E}-07$ & $7.77 \mathrm{E}-01$ & $1.65 \mathrm{E}+06$ & 1284.174 & $1.63 \mathrm{E}+04$ \\
\hline 0.5 & $2.38 \mathrm{E}+01$ & $1.86 \mathrm{E}+01$ & $1.06 \mathrm{E}-06$ & $7.82 \mathrm{E}-01$ & $9.41 \mathrm{E}+05$ & 969.8687 & $2.31 \mathrm{E}+04$ \\
\hline 0.65 & $1.07 \mathrm{E}+01$ & $4.14 \mathrm{E}+00$ & $2.37 \mathrm{E}-07$ & $3.86 \mathrm{E}-01$ & $4.23 \mathrm{E}+06$ & 2055.508 & $2.20 \mathrm{E}+04$ \\
\hline 0.8 & $5.45 \mathrm{E}+00$ & $3.59 \mathrm{E}-01$ & $2.05 \mathrm{E}-08$ & $6.58 \mathrm{E}-02$ & $4.88 \mathrm{E}+07$ & 6986.689 & $3.81 \mathrm{E}+04$ \\
\hline
\end{tabular}

\subsection{Dielectric Loss Factor}

All dielectric materials have two types of losses. One is a conduction loss, representing the flow of actual charges through the dielectric material. The other dielectric loss is due to the movement or rotation of the atoms in an alternating field. The dielectric loss factor $\varepsilon$ " with variation in frequency is as shown in Fig.2. It is considered to be the most important part of the total core loss in ferrites. The dielectric loss pattern is seen to be similar to that of dielectric constant. This dielectric loss factor is attributed to domain wall resonance. At higher frequencies, losses are found to be low, if domain wall motion is inhibited and magnetization is forced to change by rotation. The dielectric loss obtained for the present nano-ferrites synthesized by solid-state method possess lower value for $\mathrm{x}=0.8$ which makes this ferrite to be useful in the higher frequency applications like DRAs.

\subsection{Dielectric Loss Tangent}

Fig. 2(f) shows the variation of the loss tangent $\tan \delta$ with frequency for the investigated composition. It is known that loss tangent $\tan \delta$ is defined as $\varepsilon^{\prime \prime} \cdot / \varepsilon^{\prime}$. The occurrence of peaks in the variation of loss tangent with frequency can observed when the hopping frequency of localized charge carriers is approximately equal to that of the externally applied electric field; i.e., resonance phenomena. From the Fig.2 (f) it can be seen that between $\mathrm{x}=0$ and $0.8 \tan \delta$ shows a maximum at frequency between 10 and $50 \mathrm{kHz}$. Iwauchi [12] reported a strong correlation between the conduction mechanism and the dielectric behaviour of the ferrites. The conduction mechanism in n-type ferrites is considered as due to hopping of electrons between $\mathrm{Fe}^{2+}$ and $\mathrm{Fe}^{3+}$ resulting in local displacements which determines the polarization behaviour of the ferrites [13]. The condition for observing a maximum in $\tan \delta$ of a dielectric material is given by [14]:

$$
\omega \tau \sim 1
$$

where $\tau$ is the relaxation time.

And $\omega=2 \pi f_{\max }$, where $f_{\max }$ is proportional to the hopping probability $p$. The relaxation time $\tau$ is related to the hopping probability per unit time, $p$, by an equation $\tau=1 / 2 \mathrm{p}$ or $f_{\max } \alpha \mathrm{p}$. It was found to be in the range $0.3 \times 10^{-5}-1.5 \times 10^{-5} \mathrm{sec}$. A decrease of $f_{\max }$ with increasing zinc content indicates that the hopping probability per unit time decreases continuously except for $\mathrm{x}=0.65$.

\subsection{AC Conductivity}

The ac conductivity $\sigma$ and dielectric properties $\left(\varepsilon^{\prime}\right.$ and $\left.\varepsilon^{\prime \prime}\right)$ as a function of frequency at RT for the above mentioned composition are shown in Fig.2 (a-e). It can be seen that below $1 \mathrm{MHz}, \sigma$ seems to be nearly frequency independent for all compositions. Above these frequency ranges, $\sigma$ sharply increases until it reaches a maximum value. The dispersion in $\sigma$ with frequency has been explained by Koop's theorem [15] which suggested that the ferrite compact acts as multilayer capacitor. In this model, the ferrite grains and grain 
boundaries have different properties. The effect of the multilayer condenser rise with frequency as a result the conductivity increases.
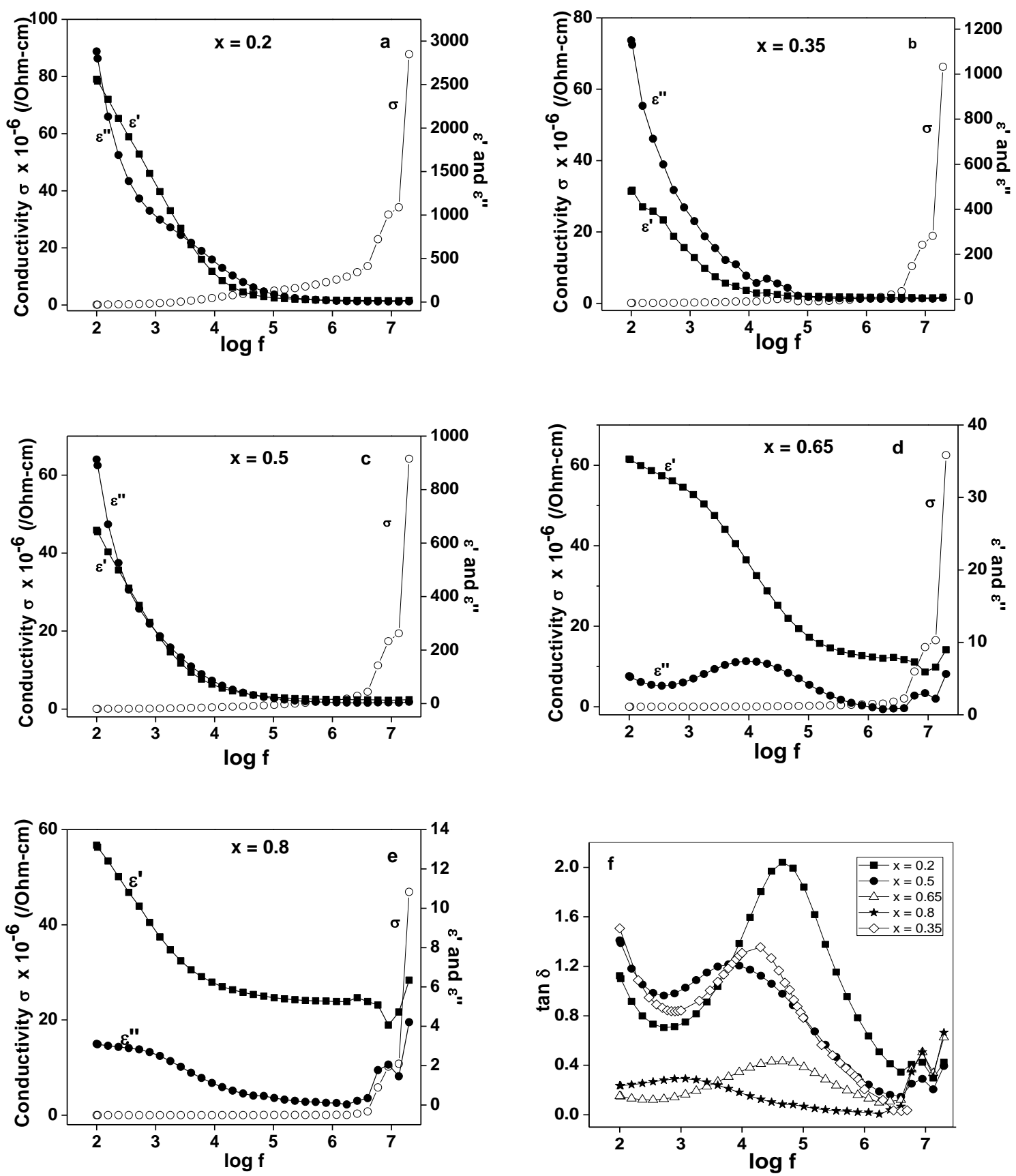

Figure 2: (a-e) plot of $\varepsilon^{\prime}, \varepsilon^{\prime \prime}$ and $\sigma$ vs. frequency and (f) $\tan \delta$ vs. frequency at room temperature
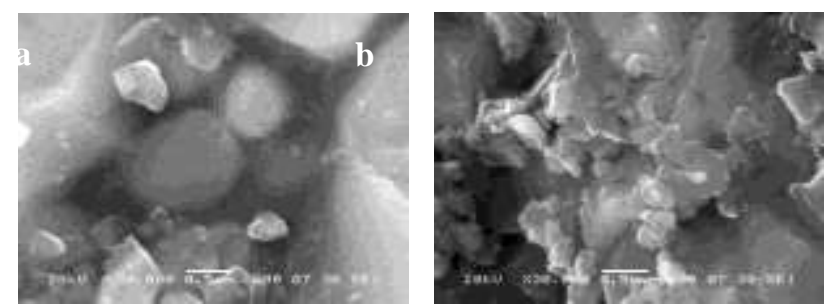

Figure 3: SEM micrographs of $\mathrm{Zn}_{\mathrm{x}} \mathrm{Mn}_{1-\mathrm{x}} \mathrm{Fe}_{2} \mathrm{O}_{4}$ samples for (a) $\mathrm{x}=0.2$ and (b) $\mathrm{x}=0.8$ 


\subsection{Relation Between the Dielectric Constant and the Resistivity}

The values of resistivity $\rho, \rho^{1 / 2}$ and $\varepsilon^{\prime} * \rho^{1 / 2}$ are shown in Table 1 . It can be seen from the table that the $\varepsilon^{\prime}$ is roughly inversely proportional to the square root of resistivity and the product $\varepsilon^{\prime} * \rho^{1 / 2}$ remains nearly constant as shown in the Table 1. A similar relationship between $\varepsilon$ and $\rho^{1 / 2}$ was found by Ravinder [16] for different ferrites. The dielectric losses in ferrites are generally reflected in the resistivity measurements, materials with low resistivity exhibiting high dielectric losses and vice versa [17]. Fig. 4 shows that the plot of dielectric constant $\varepsilon$ ' vs. zinc content $\mathrm{x}$ is an inverse image of that of $\log$ of resistivity vs. zinc content. This is a confirmation of the correlation between dielectric constant and resistivity [13].

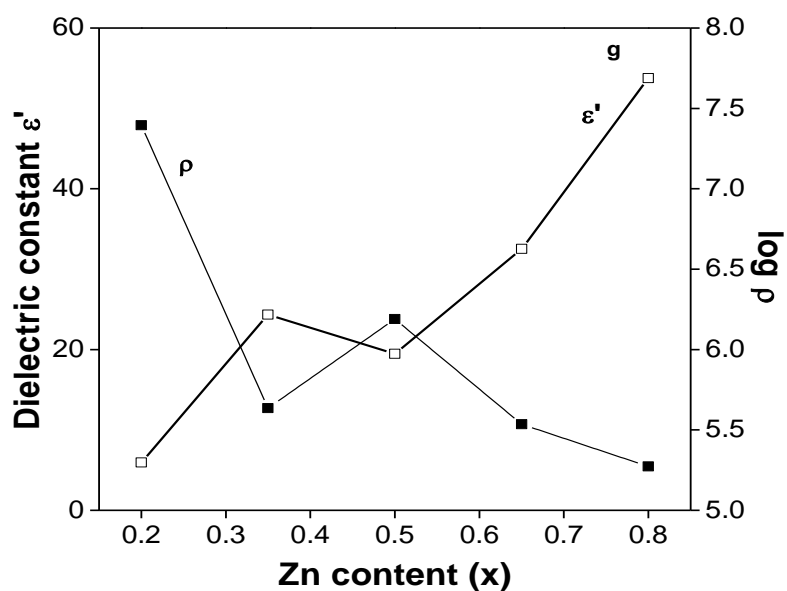

Figure 4: Plot of dielectric constant and resistivity vs Zn concentration

\section{CONCLUSION}

The dielectric properties of $\mathrm{Mn}-\mathrm{Zn}$ ferrites were investigated in this study and the results are summarized as follows:

1. The general trend for all composition is that $\varepsilon$ ' and $\varepsilon$ "' decrease with increasing frequency.

2. The value of the dielectric constant for the sample when $x=0.2$ is found to be high due to space charge polarization mechanism or could be due to morphologically clean grain structure.

3. The value of the dielectric constant for the sample when $\mathrm{x}=0.8$ is found to be low which makes this ferrite to be useful in the higher frequency applications like DRAs.

4. The frequency dependence of dielectric loss tangent $(\tan \delta)$ shows the existence of broad peaks at low frequency range.

5. The $\varepsilon$ ' is roughly inversely proportional to the square root of resistivity and the product $\varepsilon \square \rho^{1 / 2}$ remains nearly constant.

\section{ACKNOWLEDGEMENT}

Author (SMB) is thankful to University Grants Commission (UGC), New Delhi for providing financial assistance. Authors are grateful to Dr. Jyoti Jog and Mr. Chetan Chanmal, National Chemical Laboratory, Pune for providing the dielectric data of the samples.

\section{REFERENCES}

[1] Y. Chen, J. E. Snyder, C. R. Schwichtenberg, K. W. Dennis, R. W. McCallum, D. C. Jiles, Metal bonded Co-ferrite for torque sensor applications, IEEE Transactions on Magnetics, 35, 1999, 3652-3654.

[2] N. Iftimie, E. Rezlusu, P. D. Popa, N. Reslescu, Gas sensitivity of nano-crystalline nickel ferrite, Journal of Optoelectronics and Aadvance Materials, 8, 2006, 1016-1018.

[3] M. Sugimoto, The past, present and future of ferrites, Journal of American Ceramics Society, 82, 1999, 269-280.

[4] R. Lebourgeois, C. Coillot, Mn-Zn ferrites for magnetic sensor in space application, Journal of Applied Physics, 103, 2008, 07E510513.

[5] P. B. A. Fechine, R. S. T. Moretzsohn, R. C. S. Costa, J. Derov, J. W. Stewart, A. J. Drehman, C. Junqueira, A. S. B. Sombra Dielectric ferrite resonator antennas, Microwave and Optical Technology Letters, 50, 2008, 2852-2857.

[6] H. P. Klug and L. E. Alexander, X-ray Diffraction Procedures for Polycrystalline and Amorphous Materials ( John Wiley, New York, 1974) 491.

[7] D. J. Kim, Lattice parameters, ionic conductivities and solubility limits in fluorite-structure $\mathrm{MO}_{2}$ oxide $\left[\mathrm{M}=\mathrm{Hf}^{4+}, \mathrm{Zr}^{4+}, \mathrm{Ce}^{4+}, \mathrm{Th}^{4+}\right.$, $\left.\mathrm{U}^{4+}\right]$ solid solutions, Journal of American Ceramics Society, 72, 1989, 1415-16.

[8] H.M. Abdelmoneim, Dielectric properties of $\mathrm{Ti}_{x} \mathrm{Li}_{1-x} \mathrm{La}_{0.1} \mathrm{Fe}_{1.9} \mathrm{O}_{4}$ ferrite thin films, Indian Jornal of Pure and Appied. Physics, 48, $2010,562-570$

[9] G. Ranga Mohan, D. Ravinder, A.V. Ramana Reddy and B.S. Boyanov, Dielectric properties of polycrystalline mixed nickel-zinc ferrites, Materials Letters, 40, 1999, 39-45. 
[10] D.El. Kony, Dielectric Relaxation in Al-substituted Ni-Cd spinel ferrites, Egyptcian Journal of Solids, 27, 2004, 285-297.

[11] R.R. Heikes and D. Johnston, Jornal of Chemical Physics, 26, 1957, 582.

[12] K. Iwauchi, Journal of Applied Physics, 10, 1960, 1520.

[13] L.I. Rabinkin and Z.I. Novika (Ferrites Minsk, 1960) 146

[14] M. B. Reddy and P.V. Reddy, Journal of Physics D: Applied Physics, 24, 1991, 975.

[15] C.G. Koops, On the dispersion of resistivity and dielectric constant of some semiconductors at audio frequencies, Physical Review, 83, 1951, 121-124.

[16] D. Ravinder, Dielectric behaviour of lithium-cadmium ferrites, Physica Status Solidi A, 129, 1992, 549-554.

[17] A.S. Hudson, Marconi Review, 37, 1968, 43. 